\title{
PERSPECTIVE
}

\section{The safety of laser pointers: myths and realities}

\author{
John Marshall
}

Over the past few months an increasing number of reports have appeared in the popular press, describing incidents in which individuals claim to have suffered eye injury as a result of misuse of laser pointers. ${ }^{1}$ Such reports usually include a degree of media hype, together with descriptions of unlikely symptoms from indignant victims. The claims and counterclaims of occupational health and environmental safety agencies unfortunately do not attract the same degree of media interest or coverage. Motivation for the claims of individual victims are varied and include fear, ignorance, and perhaps avarice. Past experiences have shown that many individuals are truly fearful of the potential consequences of having momentarily viewed a laser beam and, therefore, seek professional advice or counsel. Other individuals are ignorant of any potential health effects, but are moved to seek advice by the group counsel of colleagues. Finally, some individuals may entertain the hope that litigation could give rise to large sums of money in settlement of personal injury claims. Given both the confusion generated by media coverage and the resultant demand on ophthalmic services in accident and emergency units, it may be helpful to review laser pointers and their interactions with ocular tissues, together with the potential confusions in international laser safety criteria.

In all laser safety documents the eye is of prime interest for two reasons. Firstly, the eye is the only organ of the body that allows optical radiations, from 400 to $1400 \mathrm{~nm}$, to penetrate deep within it. Secondly, the refractive properties of the cornea and lens result in an increase in irradiance $\left(\mathrm{W} / \mathrm{cm}^{2}\right)$ in the passage of optical radiation between the cornea and the retina of up to $10^{5}$. This increase in irradiance means that a laser achieving an irradiance of $1 \mathrm{~W} / \mathrm{cm}^{2}$ at the cornea would have an irradiance of $10 \mathrm{~kW} / \mathrm{cm}^{2}$ at the retina. If sufficient energy falls upon the retina to produce irreversible damage then such damage may occur as a result of one of three primary damage mechanisms. ${ }^{2}$ At threshold, the predominating mechanisms are roughly related to the pulse length of the laser exposure. Thus, if enough photons reach the retina to cause damage in a nanosecond or less then such damage results from ionisation or plasma formation. If it takes a few microseconds to 10 seconds to get sufficient energy at the retina to produce damage then thermal mechanisms predominate. Finally, if 10 seconds or more are required to produce change then photochemical mechanisms become involved. For exposures in the 10 second domain wavelength is of paramount importance, with shorter wavelengths (blue and ultraviolet) causing damage at lower irradiances than those in the red and infrared. It should also be remembered that while optical radiation between 400 and $1400 \mathrm{~nm}$ is refracted in a similar fashion, only those wavelengths between 400 and $780 \mathrm{~nm}$ are perceived as the sensation of light. It is this complexity of interdependence of damage induction mechanisms with wavelength, pulse duration, and energy that renders codes of practice for laser safety such complicated documents.
The earliest codes of practice, ${ }^{3}$ for the safe use of lasers, appeared within 5 years of the demonstration of the first laser in $1960 .{ }^{4}$ In the United Kingdom, ${ }^{5}$ Europe, and the United States, ${ }^{6}$ codes of practice are now published by respective standards organisations. These codes of practice have resulted in an excellent track record with relatively few laser accidents in comparison with the millions of lasers deployed worldwide. All codes of practice are based upon empirical data recording actual damage thresholds in animal studies. ${ }^{7}$ The location of damage is wavelength dependent with ultraviolet B (280-315 nm) and C (200-280 nm) and, to a lesser extent, A (315-400 nm), together with infrared B (1400-3000 nm) and C (3000-10 $000 \mathrm{~nm}$ ) being absorbed in the cornea and sclera. The portion of ultraviolet A (315-400 nm) that is transmitted by the cornea is almost completely absorbed in the lens while visible $(400-780 \mathrm{~nm})$ and infrared A (780$1400 \mathrm{~nm}$ ) are absorbed in the retina. In threshold studies data were collected which related to the $50 \%$ probability of causing a lesion in a given tissue for a given wavelength, pulse duration, and energy level. The most important data set described these so called $\mathrm{ED}_{50}$ (median effective dose) figures for irreversible retinal damage to the retina. Using these data, safety factors were then added to derive maximum permissible exposure levels (MPEs) which it is believed an individual could be exposed to without incurring injury. ${ }^{56}$ An MPE may therefore be considered as a maximum safe level of exposure. In codes of practice for the safe use of lasers MPEs are specified for the eye and the skin and vary as a function of wavelength and duration of exposure. The codes also use the MPEs to calculate a second factor, the accessible emission limit (AEL) which is the maximum value of accessible laser radiation to which an individual may get access during the operation of a given laser and remain safe. ${ }^{56}$

It should be remembered that all codes of practice are based upon the same experimental database, with major inputs from laboratories in the United States, United Kingdom, and Germany. ${ }^{7-9}$ Until relatively recently there was very good agreement throughout the world in the ways in which codes of practice utilised empirical data in deriving safety criteria; however, a dichotomy now exists between Europe ${ }^{5}$ and the United States. ${ }^{6}$ There is general agreement that to simplify laser safety manufacturers have a responsibility to indicate the magnitude of potential hazards of a given laser product by classification. Very simply, lasers are classified into four main classes of hazard, with one class being subdivided.

Class 1 systems are deemed eye safe as the AEL of such systems is always below the MPE. They have a maximum radiant emission of $0.4 \mu \mathrm{W}$ and, even if viewed over long periods of time, would produce no sustained biological change.

Class 2 systems must emit visible radiation (400-780 $\mathrm{nm}$ ) and have an upper limit of output power of $1 \mathrm{~mW}$. These systems are eye safe by virtue of the fact that if they 
fall upon the eye they are sufficiently bright that exposed individuals will blink and turn their heads away before sufficient energy has entered the eye to cause irreversible change.

Class 3 systems are divided into $3 \mathrm{~A}$ and 3B, with upper limits of emission of $5 \mathrm{~mW}$ and $500 \mathrm{~mW}$ respectively. Both European and United States codes of practice have in common a requirement that $3 \mathrm{~A}$ systems emit visible radiation and have an upper limit of emission of $5 \mathrm{~mW}$. However, in European codes there is an additional requirement that the emitted beam must not exceed an irradiance of $25 \mathrm{Wm}^{2}$. This irradiance requirement demands that beams from $3 \mathrm{~A}$ systems classified under the European system must be sufficiently divergent that an insufficient amount of the radiant emission of the laser may pass through a $7 \mathrm{~mm}$ aperture (the pupil) to exceed the limiting irradiance. Even with this difference, both European and US codes deem $3 \mathrm{~A}$ systems to be safe, in that blinking and aversion responses will ensure that insufficient energy can enter the eye to cause irreversible damage. This difference in classification has fundamental implications for laser pointers.

Class $3 B$ systems have the potential to cause significant eye damage.

Finally, class 4 systems have a radiant emission in excess of $500 \mathrm{~mW}$ and are capable of causing serious irreversible damage. All ophthalmic surgical lasers are class 4 systems.

Laser pointers may be class 1 , class 2 , or class $3 \mathrm{~A}$, using the American classification; or class 1 or 2, using European codes of practice. Class $3 \mathrm{~A}$ laser pointers classified under the American system will become 3B lasers under the European system, because they fail the irradiance test. If systems are designed to meet the European class 3A requirements the beam spreads so rapidly that the spot viewed on a screen would be so large that it would be unhelpful as a pointer. It is this differential between European and American approaches that has led to some of the media hype indicating that laser pointers fail safety tests, or exceed the levels indicated in codes of practice. ${ }^{10}$ It should be remembered that few laser pointers are manufactured in Europe and the majority come from the Pacific Rim and the United States. It should also be remembered that the major world market for laser pointers lies within the United States and outside the European Union. It is hardly surprising, therefore, that products which have the correct classification on leaving the point of manufacture may have an inappropriate classification on entering the European Union. In practice, class $3 \mathrm{~A}$ systems classified using the American standard have an upper limit of emitted power of $5 \mathrm{~mW}$, and remain safe, because of the blink reflex and aversion response. Clinically, between 100 and $500 \mathrm{~mW}$ (of emitted power) are required at diode wavelengths for retinal photocoagulation. ${ }^{112}$

On an international basis a number of agencies are concerned with the acquisition and promulgation of laser safety data. The World Health Organisation (WHO) has published a number of documents concerned with the effects of non-ionising radiation in general, and lasers in particular, ${ }^{13}$ as has the International Committee for Non-ionising Radiation Protection (ICNRP).${ }^{14}$ Such agencies feed information, together with those derived from national organisations, such as the National Radiological Protection Board (NRPB), into international standards agencies, such as the International Electro-technical Commission (IEC). These international agencies coordinate the endeavours of national standards bodies, such as the British Standards Institute (BSI). In the current British standard $^{5}$ there is a manufacturers' section in which there is mandatory compliance-that is, manufacturers must conform to standard. There is also a users section which is not mandatory and for information purposes. While not being mandatory, it should be emphasised that in any ensuing litigation the courts tend to reinforce BSI recommendations. The NRPB also has an advisory role within the United Kingdom to government departments which may have roles in enforcing laser safety criteria, such as the Home Office (HO), the Health and Safety Executive (HSE), the Department of Trade and Industry (DTI), and the Department of Health (DoH). The DTI is the agency responsible for policing laser standards in the market place, via the trading standards officers. Thus, if a laser device is thought not to comply with recommendations, the trading standards officers may seek its withdrawal. In the work environment, the HSE has a responsibility to ensure that safe procedures are carried out, via environmental health officers. These individuals may liaise within industrial organisations with the companies' own laser safety officers. In hospitals within the United Kingdom there is a shared responsibility for the safe use of lasers, between the HSE and the DoH. There is also special legislation for the use of lasers in private clinics, and these devices are governed by the Nursing Homes Act, which is enforced by local health authorities. In the public environment, the enforcement of public safety in general, and laser safety in particular, falls to the police. Again, the diversity of agencies involved in generating laser safety has led to confusion in the press, as to responsibility and blame for incidents in the general environment.

Early laser pointers were usually class 2 systems and used helium-neon laser sources with an emission wavelength at $632.8 \mathrm{~nm}$. These systems were often connected to a mains power supply, or had a limited battery life and, by definition, had an upper limit of emitted power of $1 \mathrm{~mW}$. These sources appeared extremely bright on viewing, as the $632.8 \mathrm{~nm}$ emission line was relatively close to the centre of the spectral sensitivity curve of the eye. ${ }^{15}$ More recently, laser pointers, laser pens, and key ring attachments use GaAlAs diode sources emitting at around 670 $\mathrm{nm}$. As the eye is relatively insensitive to this longer wavelength, higher powers are required to achieve the perceived brightness of a $1 \mathrm{~mW}$ helium-neon source. It is this lack of sensitivity of the eye that has resulted in manufacturers of diode based pointers having to increase the emitted power to $5 \mathrm{~mW}$. If shorter wavelengths were used, then perceived brightness would increase and radiant power could be decreased. For example, a yellow green pointer, operating at $555 \mathrm{~nm}$ and a power of $0.12 \mathrm{~mW}$, would have the same apparent brightness as a $670 \mathrm{~nm}$ red diode with a radiant power of $4 \mathrm{~mW}$. Recently, green laser pointers have become available in the United States and could be manufactured such that they would be class 2 systems and therefore eliminate the problems of current laser pointers.

In calculating MPEs, codes of practice always assume worst case conditions. ${ }^{5}$ The eye is always assumed to be perfectly refracted for the insulting wavelength, the pupil is always assumed to be $7 \mathrm{~mm}$, and although most individuals will blink and turn their head within 1/10th second of irradiation the codes of practice always assume that the aversion response will take at least $1 / 4$ second to occur. In addition to these worst case assumptions, further safety factors are incorporated in the MPEs. With all these additional factors it is, therefore, not surprising that few laser accidents have been reported, and those that have been verified occur to individuals who have been exposed to laser radiation several orders of magnitude above the MPE. Most laser accidents have occurred in research, including university, industrial institutions, or military situations where workers have been exposed to high power pulsed YAG systems operating in the infrared A. ${ }^{15-17}$ 
Laser pointers were designed to be used in lecture theatres or boardrooms, and the intention was that the operator would only activate the system when indicating points of interest in visual display material. In practice, many speakers forget that they have activated the system and turn to face the audience. The audience is usually sitting, and any exposure is likely to be transient. If a speaker persists in such careless behaviour members of the audience will almost certainly remind him of his responsibilities. Laser use by the general consumer is not governed by the discipline of the lecture theatre. Police and press report incidents whereby laser pointers have been deliberately aimed at the eyes of members of the public. This is unacceptable behaviour, but it will not result in irreversible retinal damage. Consider the difficulties of constantly placing a $5 \mathrm{~mm}$ laser spot within the pupil of a target individual. This task would be difficult, if not impossible, even in the absence of a blink reflex and aversion response in an individual who was walking. It would be absolutely impossible to sustain target fixation in an individual driving a motor vehicle. Consider further that the pupillary response would be activated on illuminating the globe, and rapidly followed by blink and aversion responses.

When the eye perceives bright sources of light one of four reactions may take place depending upon retinal irradiance. These reactions are, in order of increasing brightness: dazzle, "after image" formation, flash blindness, and irreversible damage. ${ }^{18}{ }^{19}$ The first three of these reactions are maximal in dark adapted eyes, or under night viewing conditions. Most individuals will have experienced dazzle as a result of oncoming car headlights at night and many will have noticed a temporary disturbance of vision as a result. Again, most individuals will have experienced persistent after images from accidentally staring at the filament of an incandescent bulb, or photographic flash. The after image of the source filament will again distort vision for a period, typically of some seconds to some minutes, before fading and being lost. Flash blindness is a less common phenomenon, but can occur when viewing extremely intense sources at night. Typically, flash blindness will cause visual disorientation for up to a few minutes. This phenomenon was made use of in the Falklands war when the Royal Navy deployed $20 \mathrm{~W}$ argon laser systems to "flash blind" Argentinian pilots and cause them to abort bombing missions. ${ }^{20}$ Obviously, if an individual experiences extreme dazzle, or flash blindness when driving a motor vehicle, train, or aircraft at the time of exposure, the consequences could be dire, but the laser pointer light induced eye changes will certainly not be permanent. If even higher irradiances are allowed to reach the retina then irreversible damage may ensue from ionising thermal or photochemical mechanisms.

For the general public, visual performance is poorly understood. As most individuals have good fields of view, there is an assumption that visual performance is constant over the entire area. Few lay observers understand the concept of high acuity vision and the role of the fovea in sustaining such performance. Given that the fovea is $150 \mu \mathrm{m}$ in diameter, one laser burn in this region could deprive the recipient of reading vision in that eye and, therefore, render it legally blind. By contrast, it should be remembered that thousands of burns in the periphery of a diabetic rarely give rise to visual effects detectable by the patient. It is, however, an unfortunate tenet of visual physiology that bright, or flashing, sources in the environment attract foveal fixation. The literature on accidental exposure to lasers demonstrates quite clearly that unless individuals have received damage in the macular region they are unlikely to report a suspected overexposure, and screening programmes over the past 30 years have not detected an occurrence of damage not previously subjectively detected by the victim. ${ }^{15}$

While many claims have been made for ocular and other injury, as a result of exposure to laser pointers, none has been sustained. ${ }^{21}$ Most victims are unaware of the clinical symptoms that would be appropriate to retinal injury; thus many individuals claim to have seen a bright light followed by pain or irritation in the irradiated eye. Some also complain of redness of the eye and headaches. In reality, there are no pain receptors in the retina and, therefore, threshold laser strike in the visible region of the spectrum is not associated with pain. The exception to this observation is high energy exposure to class 4 systems emitting in the red or infrared region of the spectrum and resulting in significant conduction of heat to the underlying choroid. Reddening of the eye and, less commonly, subconjunctival haemorrhages may be generated by rubbing the eyelids and thus are a secondary response caused by the patient and not the laser strike. At the wavelengths currently used for laser pointers insufficient energy will be absorbed on the skin, cornea, or sclera to produce any detectable effect. Headaches are not associated with laser damage, although may result from the individual's concern about the incident. The headaches sometimes reported subsequent to panretinal ablation seem to result from hundreds of exposures through a dilated pupil to irradiances several orders of magnitude above those associated with laser pointers. If irreversible damage had occurred, the victim would have experienced a bright flash followed by a prolonged after-image, which may be green, or red, or alternating between the two. The individual would then notice that the after image did not fade and they would be left with an area over which they could not see clearly. Scotoma of this kind would only be apparent if the causal beam had impacted upon the fovea. With peripheral lesions, as the after image faded, any underlying area of visual loss would not necessarily be detected either by the victim, or by ophthalmic examination.

If individuals present, claiming that they have been exposed to a laser pointer, they should be questioned concerning the existence of a persistent after image or scotoma. They should also be questioned about their reading vision, and it should be tested. In the absence of a persistent after image, or problems with reading vision, it is unlikely that retinal damage has been sustained and the individual should be reassured and sent home. ${ }^{22}$ While not unsafe, pupillary dilatation and fundus examination will present the individual's retina with a far higher intraocular energy load than that associated with a laser pointer and, therefore, are not recommended unless central vision has been compromised. On returning from the clinic after such an examination the individual may have photophobia, due to the dilated pupil, and/or surface irritation as a result of rubbing the irradiated eye. The patient may falsely attribute these phenomena to the laser exposure and not the examination. Misinformation given by uninformed medical personnel may add to the anxiety of the individual and is unhelpful.

In conclusion, laser pointers, pens, or key rings if used appropriately are not an eye hazard, and even if used inappropriately will not cause permanent eye damage.

JOHN MARSHALL

Department of Ophthalmology, UMDS, St Thomas's Hospital, London SE1 7EH

\footnotetext{
1 Eye experts dismiss fears of laser pens as hysteria. The Times 199724

2 Marshall J. Lasers in ophthalmology: The basic principles. Eye 1988;2 (Suppl): s98-112.

3 Laser systems: code of practice. London: Ministry of Aviation, 1965.
}

3 Laser systems: code of practice. London: Ministry of Aviation, 1965.
Mainan T. Stimulated optical radiation in ruby lasers. Nature 1960;187:493. 
5 BSEN 60825: 992. Radiation safety of laser products, equipment, classification requirements and users guide. London: British Standards classification, requir.
Institution, 1992 .

6 American National Standards Institute. American National Standard for the safe use of lasers, ANSI Z136.1, New York: National Standards Institute, 1993.

7 Borland RC, Brennan DH, Marshall J, et al. The role of fluorescein angiography in the detection of laser-induced damage to the retina: a threshold study for Q-switched, neodymium and ruby lasers. Exp Eye Res 1978;27:471-93.

8 Beatrice ES, Fisch GD. Retinal laser damage thresholds as a function of image diameter. Arch Environ Health 1973;27:322-6.

9 Goldman AL, Ham WT, Mueller HA. Ocular damage thresholds and mechanisms for ultrashort pulses of both visible and infrared laser radiation in rhesus monkey. Exp Eye Res 1977;24:45-56.

10 McGhee CNJ, Craig JP, Moseley H, et al. Laser key chains: potential for serious eye injury. Eye News 1997/8;4:17-19.

11 McHugh JDA, Marshall J, ffytche TJ, et al. Initial clinical experience using a diode laser in the treatment of retinal vascular disease. Eye 1989;3:516-27.

12 McHugh JDA, Marshall J, ffytche TJ, et al. Macular photocoagulation of human retina with a diode laser: A comparative histopathological study. Lasers Light Ophthalmol 1990:3:11-28.
13 World Health Organisation. Environmental Health Criteria 23, Lasers and optical radiation Geneva: WHO, 1983

14 Jammet HP, Bosnjakovic BFM, Czerski P, et al Guidelines on limits of exposure to laser radiation of wavelengths between $180 \mathrm{~nm}$ and $1 \mathrm{~mm}$. Health Physics 1985;49:341-59.

15 Sliney DH, Wolbarsht ML. Safety with lasers and other optical sources. New York: Plenum Press, 1986.

16 Gabel VP, Birngruber R, Lorenz B. Clinical observations of six cases of laser injury to the eye. Health Physics 1989;56:705-10.

17 Al Halel A, Glovinsky Y, Treister G. Long term follow up of accidental parafoveal laser burns. Retina 1993;13:152-4.

18 International Committee of the Red Cross. Blinding weapons. Geneva: ICRC, 1993

19 Mellerio J, Marshall J, Tengroth B, et al. Battlefield laser weapons: an assessment of systems, hazards, injuries and ophthalmic resources required for treatment. Lasers Light Ophthalmol 1991;4:41-67.

20 Gallego F, Daly M. Laser weapons in Royal Navy service. Fane's Defence Weekly 199013 January.

21 Mainster MA, Timberlake GT, Warren KA, et al. Pointers on laser pointers. Ophthalmology 1997;104:1213-14.

22 Mensah E, Vafidis G, Marshall J. Laser pointers: the facts, media hype and hysteria. Lancet 1998; (in press)

\section{Contributors please note:}

Communications from the UK, Republic of Ireland, Australasia, Asia, Africa, Japan, and the Middle East should be sent to the Editor, British Journal of Ophthalmology, Department of Ophthalmology, University of Aberdeen, Medical School, Foresterhill, Aberdeen AB25 2ZD, Scotland, UK (Tel: 01224 663812; Fax: 01224 663832).

Communications from Western Europe, Eastern Europe, the former Soviet Union, and Scandinavia should be sent to Professor A Kijlstra, Associate editor, The Netherlands Ophthalmic Research Institute, PO Box 12141, Meibergdreef 9, 1100 AC Amsterdam, Netherlands (Tel: +31 20 5664535; Fax: +31 20 6913401).

Communications from the USA, Canada, and South America should be sent to Professor C Hoyt, Associate editor, University of California, UCSF Ophthalmology Department, Pediatric Ophthalmology, 400 Parnassus Avenue, Room 702 A, Box 0344, San Francisco, CA 94143, USA (Tel: +415 476 1289/1921; Fax: +415 502 6468). 